\title{
Log odds of positive lymph nodes is an excellent prognostic factor for patients with rectal cancer after neoadjuvant chemoradiotherapy
}

\author{
Tianlei Xu ${ }^{1,2}$, Lin Zhang ${ }^{1,2}$, Liang Yu ${ }^{1,2}$, Yuelu Zhu ${ }^{3}$, Hui Fang ${ }^{4}$, Bo Chen ${ }^{4}$, Haizeng Zhang ${ }^{1,2}$ \\ ${ }^{1}$ Department of Colorectal Surgery, National Cancer Center/National Clinical Research Center for Cancer/Cancer Hospital, Chinese Academy \\ of Medical Sciences and Peking Union Medical College, Beijing, China; ${ }^{2}$ State Key Laboratory of Molecular Oncology, National Cancer Center/ \\ National Clinical Research Center for Cancer/Cancer Hospital, Chinese Academy of Medical Sciences and Peking Union Medical College, Beijing, \\ China; ${ }^{3}$ Department of Pathology, National Cancer Center/National Clinical Research Center for Cancer/Cancer Hospital, Chinese Academy \\ of Medical Sciences and Peking Union Medical College, Beijing, China; ${ }^{4}$ Department of Radiation Oncology, National Cancer Center/National \\ Clinical Research Center for Cancer/Cancer Hospital, Chinese Academy of Medical Sciences and Peking Union Medical College, Beijing, China \\ Contributions: (I) Conception and design: T Xu, L Zhang, H Zhang; (II) Administrative support: Y Zhu, H Fang, B Chen, T Xu, H Zhang; (III) \\ Provision of study materials or patients: T Xu, L Yu, H Zhang; (IV) Collection and assembly of data: T Xu, L Yu, B Chen, H Zhang; (V) Data analysis \\ and interpretation: T Xu, L Zhang, Y Zhang, H Fang, H Zhang; (VI) Manuscript writing: All authors; (VII) Final approval of manuscript: All authors. \\ Correspondence to: Haizeng Zhang, MD. State Key Laboratory of Molecular Oncology, Department of Colorectal Surgery, National Cancer Center/ \\ National Clinical Research Center for Cancer/Cancer Hospital, Chinese Academy of Medical Sciences and Peking Union Medical College, Beijing \\ 100021, China. Email: haizengzhang@cicams.ac.cn.
}

Background: Neoadjuvant chemoradiotherapy (NCRT) results in fewer lymph nodes harvested and causes staging migration. Therefore, we compared the prognostic value of the logarithmic odds of positive lymph nodes (LODDS) with the lymph node ratio (LNR) and the American Joint Committee on Cancer (AJCC) ypN stage in patients with locally advanced rectal cancer (LARC) after NCRT.

Methods: A total of 445 patients with LARC who received NCRT and underwent radical surgery between January 2004 and December 2015 were recruited, and data from 4881 patients included in the Surveillance, Epidemiology and End Results (SEER) database between 2010 and 2013 were analyzed to verify our results. The time-dependent area under the receiver operating characteristic curve (TimeROC) was used to evaluate the discriminative ability of the different lymph node staging systems.

Results: ypN staging failed to satisfactorily stratify the patients treated with NCRT [the 3-year disease-free survival (DFS) rates were $65.7 \%$ and $55.4 \%$ for the ypN1 and ypN2 groups, respectively, $\mathrm{P}=0.252]$. The LODDS classification was significantly associated with DFS, and the 3-year DFS rates for the LODDS0, LODDS1, and LODDS2 groups were 89.9\%, 72.4\%, and 53.9\%, respectively ( $\mathrm{P}<0.05$ across all groups). Furthermore, the LODDS classification system was able to subclassify patients with ypN0 stage tumors regardless of whether $\geq 12$ or $<12$ total lymph nodes (TLNs) were harvested. TimeROC analysis showed that the LODDS classification (AUC, median: 0.722, range: 0.692-0.754) had a higher accuracy for determining the prognosis than the ypN stage (AUC, median: 0.691, range: 0.684-0.712) or the LNR (AUC, median: 0.703, range: 0.685-0.730) classification, regardless of lymph node status. These results were verified using the SEER database.

Conclusions: The LODDS was a better prognostic factor for DFS than ypN staging or the LNR-based approach in patients with LARC after NCRT, particularly those with <12 TLNs harvested or ypN0 stage disease.

Keywords: Logarithmic odds of positive lymph nodes (LODDS); lymph node ratio (LNR); staging; neoadjuvant chemoradiotherapy (NCRT)

Submitted Nov 21, 2020. Accepted for publication Jan 24, 2021.

doi: $10.21037 /$ atm-20-7590

View this article at: http://dx.doi.org/10.21037/atm-20-7590

(c) Annals of Translational Medicine. All rights reserved. 


\section{Introduction}

Currently, neoadjuvant chemoradiotherapy (NCRT) is the standard treatment for locally advanced mid-low rectal cancer (LARC) (1). The American Joint Committee on Cancer (AJCC) $8^{\text {th }}$ TNM staging system is currently used to determine the prognosis of patients who receive NCRT, but it employs the same categorical definitions used for patients who do not receive NCRT (2). The ypN stage is still determined according to the number of positive lymph nodes (PLNs) and is regarded as the most important prognostic factor for patients with LARC who are treated with NCRT (3). However, NCRT may result in fewer total lymph nodes (TLNs) harvested and PLNs retrieved, as well as $\mathrm{N}$ downstaging $(3,4)$. According to Mechera et al., there is a reduction in the mean number of TLNs and PLNs harvested (3.9 and 0.7, respectively) from patients treated with NCRT compared to those treated without NCRT (4). Furthermore, fewer than 12 lymph nodes are harvested after NCRT in approximately $30-50 \%$ of patients $(5,6)$. NCRT usually exerts an $\mathrm{N}$ downstaging effect due to the treatment response. Therefore, NCRT may also result in ypN stage migration, which may not provide an accurate assessment of lymph node staging $(4,7,8)$.

The lymph node ratio (LNR), which is defined as the ratio of PLNs to TLNs, has functioned as a novel prognostic factor in various cancer types $(9,10)$. Because the LNR includes information on both the PLNs and TLNs retrieved, some authors have proposed that the LNR may help to reduce the number of lymph nodes harvested and address the limitation of the ypN staging system in patients with rectal cancer after NCRT. However, the LNR does not provide a better prognostic evaluation than the ypN staging system for patients without lymph node metastases (ypN0 patients). In addition, many studies have confirmed that the number of negative lymph nodes (NLNs) significantly affects prognosis $(11,12)$. The $\mathrm{LNR}$, similar to ypN staging, does not consider the effect of NLNs on patient prognosis.

The logarithmic odds of positive lymph nodes (LODDS), which is defined as the $\log$ of the ratio of the number of PLNs to the number of NLNs (13), has recently been introduced and confirmed as a prognostic factor in colorectal cancer (CRC), pancreatic cancer and gastric cancer $(10,13,14)$. Because NCRT frequently leads to a decrease in the number of TLNs harvested and PLNs retrieved, the LODDS is estimated using a method that considers the effects of both PLNs and NLNs on prognosis. Hence, LODDS may provide a more accurate assessment of the prognosis for patients with LARC after NCRT than ypN and LNR. As shown in the study by Huang et al., LODDS shows superior power to the LNR in discriminating the cancer-specific survival (CSS) of patients with LARC and lymph node metastases after NCRT (ypN+ patients). However, this study was based on the Surveillance, Epidemiology and End Results (SEER) public database and did not include a validation cohort; in addition, it explored only the prognostic role of LODDS in ypN+ patients. Moreover, after neoadjuvant treatment, approximately $60 \%$ of the patients exhibited no lymph node metastasis, but fewer than 12 TLNs were harvested in approximately $30-50 \%$ of the patients $(15,16)$. Furthermore, patients with $<12$ TLNs harvested do not typically present with lymph node metastasis (17). The prognostic value of the LODDS for patients with rectal cancer treated with NCRT has rarely been reported, and thus, the prognostic value of the LODDS for patients without lymph node metastasis or with different numbers of harvested lymph nodes remains unclear.

In the present study, we compared the prognostic values of $y p N$, the LNR, and the LODDS in 445 patients with rectal cancer who received NCRT and underwent radical surgery, particularly patients with $<12$ TLNs harvested and those with no lymph node metastasis. We also verified the results using the data from patients included in the SEER database.

We present the study in accordance with the REMARK reporting checklist (available at http://dx.doi.org/10.21037/ atm-20-7590).

\section{Methods}

All procedures performed in this study involving human participants were in accordance with the Declaration of Helsinki (as revised in 2013). This study was reviewed and approved by the Ethics Committee of Cancer Hospital, Chinese Academy of Medical Sciences (No. NCC2019C-96). Because of the retrospective nature of the study, the requirement for informed consent was waived.

\section{Single-center patient cohort}

Four hundred forty-five consecutive patients with LARC who received NCRT and underwent radical surgery at the Cancer Hospital, Chinese Academy of Medical Sciences (CICAMS) between January 2004 and December 2015 were recruited.

The inclusion criteria were as follows: (I) biopsy- 
proven rectal adenocarcinoma, (II) a distance from the lower margin of the tumor to the anal verge less than $10 \mathrm{~cm}$, (III) clinical stage II or III tumors, (IV) long-course NCRT followed by radical excision, and (V) no evidence of distant metastatic disease noted either before surgery or during the operation. Patients with inflammatory bowel disease, N1c patients [without lymph node metastases but with extranodal tumor deposits (TDs)], or patients who developed distant metastasis within 6 months after receiving treatment were excluded. Patients who had a history of familial adenomatous polyposis or Lynch syndrome and patients with a synchronous or metachronous second tumor were excluded. Patients who participated in clinical trials or received short-term radiotherapy were excluded.

\section{SEER validation cohort}

Data from additional patients were obtained from the SEER 18 (range from 1973-2015) database released in June 2018 and analyzed using SEER*Stat 8.3 .5 software. A total of 5,565 patients with primary adenocarcinoma of the rectum treated with NCRT and underwent radical surgery between 2010 and 2013 were identified. The inclusion criteria were as follows: (I) pathologically confirmed rectal adenocarcinoma, (II) treatment with long-course NCRT and radical surgery, (III) the absence of distant metastasis at diagnosis or during surgery, (IV) the absence of a second malignant tumor, (V) availability of accurate tumor-specific survival data, and (VI) availability of detailed information regarding the pathological lymph node status. Patients with the N1c stage were excluded. In total, 4,881 patients were ultimately included in the study. In this study, CSS was defined as death caused by rectal cancer.

\section{Patient cobort stratification}

The ypN staging system is based on the $8^{\text {th }}$ TNM staging system (ypN0: no lymph node metastasis, ypN1: 1-3 PLNs, and ypN2: >3 PLNs). The LNR is defined as the ratio of the number of PLNs to TLNs retrieved (LNR = PLNs/TLNs). According to the best cutoff value of the LNR, patients were divided into three groups: LNR0: no metastasis; LNR1: $0<\mathrm{LNR} \leq 0.2$; and LNR2: $\mathrm{LNR}>0.2$. The LODDS is calculated as the log of (PLNs + 0.5)/(NLNs +0.5 ), with 0.5 added to the numerator and denominator to avoid divisions by zero. Cutoff values of -1.1 and -0.6 were used to stratify patients into three groups: LODDS0 $\leq-1.1$; $-1.1<$ LODDS1 $\leq-0.6$; and LODDS2 >-0.6. A minimum of 12 TLNs harvested is a widely acknowledged standard. Therefore, we divided the patients into two groups based on whether $<12$ TLNs or $\geq 12$ TLNs were harvested for the subgroup analysis.

\section{Treatment}

The initial pretreatment clinical stage was determined using rectal magnetic resonance imaging (MRI), endoscopic ultrasound, and chest/abdominal/pelvic computerized tomography (CT) according to the recommendations of the $8^{\text {th }}$ edition of the TNM staging system. All patients received long-course NCRT with a total dose of 40-50 (median: 50) Gy administered in 20 to 28 fractions during a 5-week period, concurrently with capecitabine $\left(825 \mathrm{mg} / \mathrm{m}^{2}\right.$, bis in die) with or without oxaliplatin (infusion $50 \mathrm{mg} / \mathrm{m}^{2}$, weekly). All patients underwent radical surgery according to the total mesorectal excision (TME) principle approximately 6-8 weeks after the completion of radiation therapy. Regardless of the surgical and pathological results, postoperative adjuvant chemotherapy was recommended for all patients.

\section{Pathological evaluation}

Standard hematoxylin-eosin and saffron staining of each paraffin block was performed for histologic examination. All slices were re-evaluated by two professional gastrointestinal pathologists. We reevaluated the depth of tumor invasion (ypT), the total number of lymph nodes harvested, the number of metastatic lymph nodes (If there is residual live tumor cells, even rare residual tumor cell in the lymph node), perineural invasion, vascular invasion, circumferential resection margin (CRM), and tumor regression grade (TRG). The TRG was evaluated according to the TRG system proposed by Mandard et al. (18).

\section{Follow-up}

NCCN guidelines recommend follow-up every 3 months for 2 years after the initial surgery, every 6 months for the next 3 years, and yearly thereafter. Postoperative followup included a physical examination, carcinoembryonic antigen (CEA) monitoring, chest/abdominal/pelvic CT, pelvic MRI, and colonoscopy. Data on whether and when the patients developed local recurrence or distant metastasis were obtained from outpatient or inpatient records or via telephone follow-up. Disease-free survival (DFS) was 


\section{Page 4 of 16}

defined as the time from the date of operation to the date of first local recurrence or distant metastasis. All patients were followed until either death or September 2018.

\section{Statistical analysis}

The associations of ypN stage, LNR, and LODDS with DFS were determined by univariate analyses. Survival analyses were performed using the Kaplan-Meier method and the log-rank test. $\mathrm{P}$ values less than 0.05 were considered statistically significant. Comparisons among the three lymph node staging systems were performed with a time-dependent area under the receiver operating characteristic curve (TimeROC). Cox proportional hazards regression analysis was performed for the multivariate analysis, and a multivariate Cox regression model was performed to jointly evaluate the prognostic capacity and other possible prognostic indicators of the three lymph node staging schemes. All statistical analyses were conducted using R software (version 3.5.1, https://www.r-project.org/).

\section{Results}

\section{Demographic and clinicopathological characteristics of the patients}

The clinical and demographic characteristics of all patients are presented in Table 1. The study population comprised 294 men $(294 / 445,66.1 \%)$ and 151 women $(151 / 445$, $33.9 \%$ ), with a median age of 55 (range: $23-81$ ) years. Among the 445 patients, 392 (88.1\%) had cT3 stage disease, $53(11.9 \%)$ had cT4 stage disease, and 197 (44.3\%) had stage $\mathrm{cN}+$ disease before NCRT. After NCRT, the majority of the patients were found to have moderately differentiated tumors: 62 patients $(13.9 \%)$ had perineurial invasion, and 20 patients $(4.5 \%)$ had vascular invasion. The prognosis was worse for the patients who exhibited perineurial invasion or vascular invasion. After surgery, 228 (51.2\%) patients received the recommended postoperative adjuvant chemotherapy. The median follow-up duration was 46.7 (range: 12.2$148.7)$ months. Fifteen (3.4\%) patients developed local recurrence, 89 (20.0\%) developed distant metastasis, and $5(1.1 \%)$ developed both local and systemic recurrence.

\section{The pathological index of the lymph nodes}

The median number of dissected TLNs was 14 (range: $0-61$ ), and the median number of PLNs was 2 (range:
Xu et al. Prognostic value of LODDS for rectal cancer after NCRT

$1-21)$. Among the 445 patients, 186 (41.8\%) had $<12$ TLNs harvested, and $291(65.4 \%)$ patients were staged as ypN0. In the ypN0 group, $44.3 \%$ (129/291) of patients had <12 TLNs harvested, and in the ypN+ group, 37.0\% (57/154) of patients had $<12$ TLNs harvested. The proportions of ypN0 patients with $<12$ and $\geq 12$ TLNs harvested were $69.4 \%$ (129/186) and $62.5 \%(162 / 259)$, respectively. DFS was significantly different between patients with $<7$ and $\geq 7$ TLNs harvested $(\mathrm{P}=0.023$, Table 1$)$ as well as between groups stratified by NLNs with a cutoff value of 10 (Table 1); however, when the TLN cutoff value was set to 12 , the difference in DFS was not statistically significant $(\mathrm{P}=0.157$, Table 1$)$.

\section{Prognostic value of $y p N$, the LNR, and the LODDS and multivariate analysis}

In the stratified univariate analysis, a significant difference in DFS was not observed between the ypN1 and ypN2 groups $(\mathrm{P}=0.252$, Figure $1 A)$. The 3 -year DFS rates were $89.3 \%, 65.7 \%$, and $55.4 \%$ for the ypN0 ( $\mathrm{n}=291)$, ypN1 $(\mathrm{n}=113)$, and ypN2 ( $\mathrm{n}=41)$ groups, respectively. However, the LNR and LODDS classification systems were significantly associated with DFS in the subgroup analysis. The 3-year DFS rates were $89.3 \%, 69.3 \%$, and $51.4 \%$ for the LNR0 ( $\mathrm{n}=291)$, LNR1 $(\mathrm{n}=102)$, and LNR2 $(\mathrm{n}=52)$ groups, respectively $(\mathrm{P}<0.05$ across all groups; Figure $1 B)$. Furthermore, the 3 -year DFS rates for the LODDS0 $(\mathrm{n}=276)$, LODDS1 $(\mathrm{n}=99)$, and LODDS2 $(\mathrm{n}=70)$ groups were $89.9 \%, 72.4 \%$, and $53.9 \%$, respectively ( $\mathrm{P}<0.05$ across all groups; Figure 1C). TimeROC analysis was performed to determine which lymph node staging systems predicted DFS most accurately. As shown in Figure 1D, the LODDS had a higher area under the receiver operating characteristic curve (AUC, median: 0.722, range: 0.692-0.754) for DFS than ypN (median: 0.691, range: $0.684-0.712$ ) or LNR (median: 0.703, range: 0.685-0.730) staging for the entire cohort of patients, indicating its better performance at determining the prognosis of patients with rectal cancer after NCRT. The multivariate analysis showed that the three models including the ypN, LNR, or LODDS classifications were capable of predicting DFS. In addition, the model including the LODDS classification presented the highest value for Harrell's C statistic (Table 2).

\section{Prognostic value of $y p N$ staging, the $L N R$, and the LODDS among the $<12$ TLN and $\geq 12$ TLN subgroups}

The ypN staging, LNR and LODDS were significantly 
Table 1 Demographic and clinicopathological characteristics of the 445 patients and univariable analysis results for 3-year DFS

\begin{tabular}{|c|c|c|c|}
\hline Variables & $\mathrm{N}(\%)$ & 3-year DFS rate (\%) & $P$ value \\
\hline Male & $294(66.1)$ & 79.5 & \\
\hline Female & $151(33.9)$ & 81.4 & \\
\hline Age (years) & & & 0.381 \\
\hline$\geq 65$ & $93(20.9)$ & 82.2 & \\
\hline Location (cm) & & & 0.707 \\
\hline Lower [0-5] & $316(71.0)$ & 79.0 & \\
\hline Middle [6-10] & $129(29.0)$ & 83.0 & \\
\hline Laparoscopic & $254(57.1)$ & 77.5 & \\
\hline Surgical procedure & & & 0.484 \\
\hline LAR & $193(43.4)$ & 80.4 & \\
\hline APR & $222(49.9)$ & 80.5 & \\
\hline Hartman & $30(6.7)$ & 71.5 & \\
\hline Clinical T stage & & & 0.962 \\
\hline cT3 & $392(88.1)$ & 80.4 & \\
\hline cT4 & $53(11.9)$ & 78.7 & \\
\hline$>5$ & $195(43.8)$ & 79.6 & \\
\hline$\leq 5$ & $203(45.6)$ & 82.8 & \\
\hline Unknow & $47(10.6)$ & 71.3 & \\
\hline Preoperative CEA, ng/mL & & & 0.482 \\
\hline$>5$ & $150(33.7)$ & 78.1 & \\
\hline$\leq 5$ & $261(58.7)$ & 79.9 & \\
\hline Unknow & $34(7.6)$ & 88.2 & \\
\hline Concurrent chemotherapy & & & 0.065 \\
\hline Capecitabine & $266(59.8)$ & 82.9 & \\
\hline Capecitabine plus oxaliplatin & $179(40.2)$ & 78.8 & \\
\hline
\end{tabular}

Table 1 (continued) 
Table 1 (continued)

\begin{tabular}{|c|c|c|c|}
\hline Variables & $\mathrm{N}(\%)$ & 3-year DFS rate (\%) & $P$ value \\
\hline$\leq 7$ & $192(43.1)$ & 79.0 & \\
\hline$>7$ & $253(56.9)$ & 81.0 & \\
\hline Adjuvant chemotherapy & & & 0.063 \\
\hline No & $217(48.8)$ & 83.7 & \\
\hline Perineurial invasion & & & 0.045 \\
\hline Positive & $62(13.9)$ & 67.4 & \\
\hline Negative & $383(86.1)$ & 91.4 & \\
\hline Well & $14(3.1)$ & 85.7 & \\
\hline Unknow & $39(8.8)$ & 78.3 & \\
\hline Intra-vascular invasion & & & $<0.001$ \\
\hline Positive & $20(4.5)$ & 48.1 & \\
\hline Negative & $425(96.5)$ & 81.6 & \\
\hline Number of retrieved TLNs & & & 0.157 \\
\hline$<12$ & $186(41.8)$ & 76.4 & \\
\hline$<10$ & $170(38.2)$ & 71.9 & \\
\hline$\geq 10$ & $275(61.8)$ & 85.2 & \\
\hline ypTNM & & & $<0.001$ \\
\hline 0 & $70(15.7)$ & 95.6 & \\
\hline I & $112(25.2)$ & 88.2 & \\
\hline ॥ & $109(24.5)$ & 87.0 & \\
\hline III & $154(34.6)$ & 63.0 & \\
\hline \multicolumn{4}{|l|}{ Follow-up } \\
\hline Local recurrence & $15(3.4)$ & & \\
\hline Systematic recurrence & $89(20.0)$ & & \\
\hline Local and systematic recurrence & $5(1.1)$ & & \\
\hline
\end{tabular}

DFS, disease-free survival; LAR, low anterior resection; APR, abdominal-perineal resection; CEA, carcinoembryonic antigen; NCRT, neoadjuvant chemoradiotherapy; TLN, total lymph node; NLN, number of negative lymph nodes. 
A

Overall patients

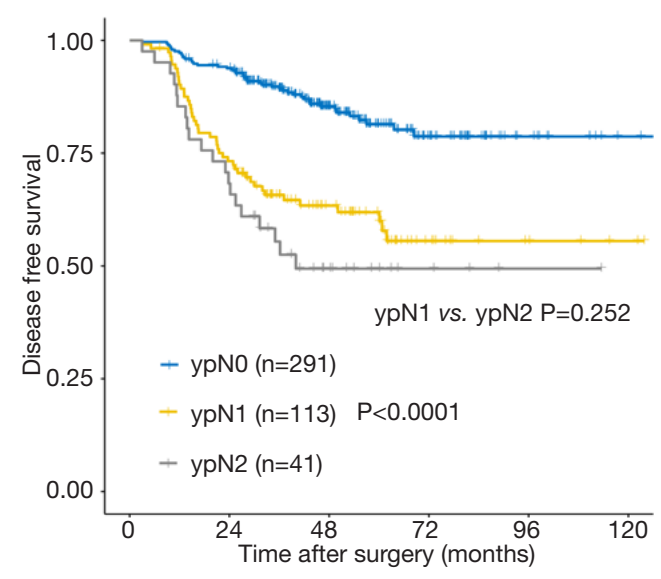

C

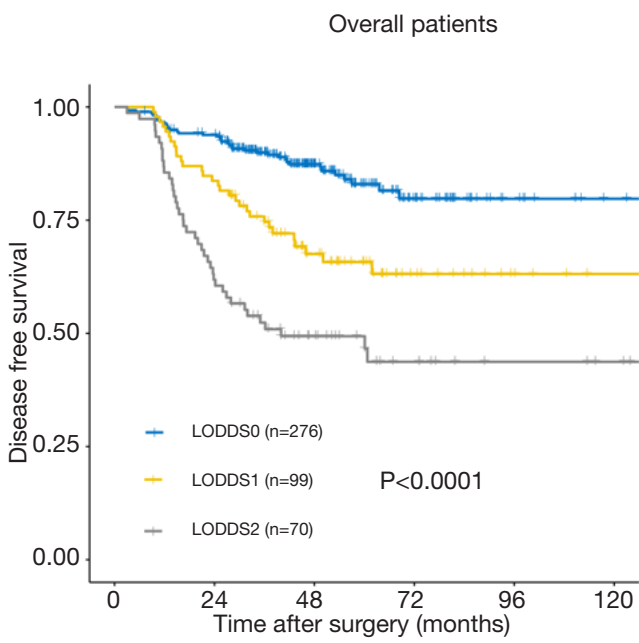

B

Overall patients
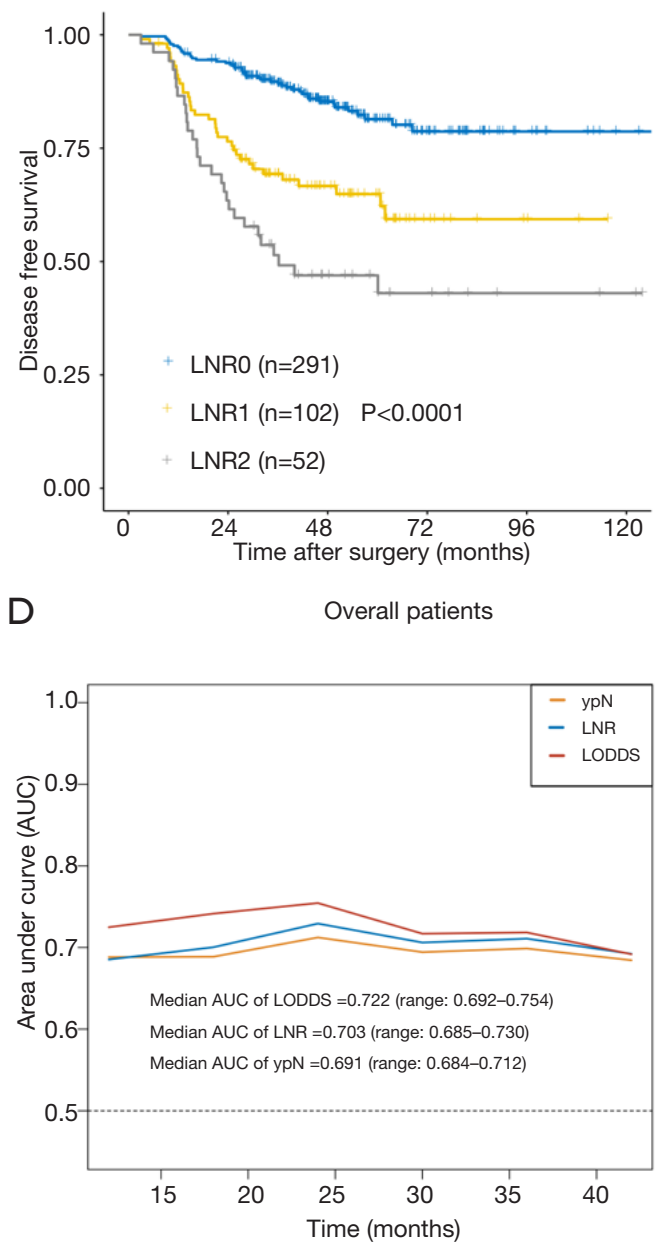

Figure 1 Kaplan-Meier analysis of DFS according to the ypN stage, LNR, and LODDS classification in all patients (A,B,C) and TimeROC analysis of ypN, LNR and LODDS in all patients (D). DFS, disease-free survival; LNR, lymph node ratio; LODDS, logarithmic odds of positive lymph nodes; TimeROC, time-dependent area under the receiver operating characteristic curve.

associated with DFS between subgroups when $\geq 12$ TLNs were harvested $(\mathrm{P}<0.05$ across all groups, Table 3). In the subgroup analysis, a significant difference in DFS was not observed between the ypN1 and ypN2 groups when $<12$ TLNs were harvested $(\mathrm{P}=0.454$, Figure $2 A)$. Similarly, the LNR was also not associated with DFS between the LNR1 and LNR2 subgroups when $<12$ TLNs were harvested $(\mathrm{P}=0.756$, Figure $2 B)$. However, the LODDS classification system was significantly associated with DFS between subgroups when $<12$ TLNs were harvested $(\mathrm{P}<0.05$ across all groups; Figure 2C), with 3-year DFS rates of $91.8 \%$, $75.1 \%$, and $49.9 \%$ for the LODDS0 ( $\mathrm{n}=98)$, LODDS 1 $(\mathrm{n}=50)$, and LODDS2 $(\mathrm{n}=38)$ groups, respectively (Table 3). As shown in Figure 2D, LODDS had a higher AUC (median:
0.762 , range: $0.720-0.832$ ) than the ypN (median: 0.717 , range: $0.697-0.746$ ) or LNR (median: 0.714 , range: $0.686-$ 0.744 ) staging in the $<12$ TLNs group. Based on these results, the LODDS classification had a higher accuracy of determining the prognosis than the ypN and LNR classifications for patients with $<12$ TLNs harvested.

\section{Prognostic value of the LODDS in the ypNO and ypN+ subgroups}

Patients with ypN0 stage disease were further subdivided according to the LODDS classification into high-risk and low-risk groups (Figure $3 A, \mathrm{P}=0.005$ ); patients in the highrisk group were found to have fewer NLNs than those in 
Table 2 Multivariable analysis of the prognostic performance of the three models with different node staging classifications

\begin{tabular}{|c|c|c|c|c|c|c|}
\hline Variables & \multicolumn{2}{|l|}{ ypN stage } & \multicolumn{2}{|c|}{ LNR classification } & \multicolumn{2}{|c|}{ LODDS classification } \\
\hline TRG & & $<0.001$ & & $<0.001$ & & $<0.001$ \\
\hline TRG1 & Reference & & Reference & & Reference & \\
\hline TRG2 & $1.363(0.584-3.184)$ & & $1.376(0.587-3.224)$ & & $1.448(0.624-3.363)$ & \\
\hline TRG4 & $2.461(1.104-5.488)$ & & $2.490(1.115-5.558)$ & & $2.761(1.253-6.085)$ & \\
\hline TRG5 & $15.803(5.010-49.851)$ & & $15.685(4.955-49.657)$ & & $17.522(5.462-56.207)$ & \\
\hline NLN & & 0.007 & & 0.015 & & NA \\
\hline$N L N<10$ & Reference & & Reference & & NA & \\
\hline ypNO (or LNR0 or LODDS0) & Reference & & Reference & & Reference & \\
\hline ypN1 (or LNR1 or LODDS1) & $2.428(1.522-3.874)$ & & $2.528(1.562-4.092)$ & & $2.261(1.397-3.661)$ & \\
\hline ypN2 (or LNR2 or LODDS2) & $3.258(1.870-5.677)$ & & $2.860(1.669-4.901)$ & & $3.417(2.051-5.694)$ & \\
\hline Harrell's C & $0.723(0.697-0.749)$ & & $0.721(0.695-0.747)$ & & $0.728(0.703-0.753)$ & \\
\hline
\end{tabular}

${ }^{a}$, three COX regression models were constructed respectively and the node stage represent the ypN, LNR and LODDS classifications for corresponding multivariable models. LNR, lymph nodes ratio; LODDS, logarithmic odds of positive lymph nodes; TRG, tumor regression grading; NLN, number of negative lymph nodes.

the low-risk group. The 3-year DFS rates were 91.0\%, $80.7 \%$, and $75.1 \%$ for the LODDS0 ( $\mathrm{n}=243)$, LODDS 1 $(\mathrm{n}=40)$, and LODDS2 $(\mathrm{n}=8)$ groups, respectively $(\mathrm{P}=0.007$; Table 4). The ypN+ stage patients were also further subdivided into a high-risk group and a low-risk group according to the LODDS classification, and significant differences in DFS were found between the subgroups ( $\mathrm{P}=0.032$, Table 4), even when we combined the LODDS0 $(\mathrm{n}=33)$ group with the LODDS1 $(\mathrm{n}=59)$ group (Figure $3 B$, $\mathrm{P}=0.004)$. TimeROC analysis showed that the AUC of the LODDS classification (median: 0.583, range: 0.539-0.669, Figure $3 C$ ) was higher than that of $\mathrm{ypN}$ staging and the LNR (AUC of ypN or LNR =0.5) in the ypN0 group. Similarly, for ypN+ stage patients, the AUC of the LODDS classification (median: 0.599, range: 0.534-0.612, Figure 3D) was higher than that of ypN staging (median: 0.535 , range: $0.522-0.554$ ) and the LNR (median: 0.573, range: $0.540-0.582$ ). Thus, the LODDS classification had better accuracy in determining the prognosis than the ypN and LNR classifications for patients with or without lymph node metastases, and the LODDS classification displayed a natural advantage in evaluating patients without lymph node metastases.

\section{Validation of the prognostic value of the LODDS, LNR, and ypN staging systems in the SEER cohort}

Among the 4,881 patients in the SEER cohort, 1,718 (35.2\%) had $<12$ TLNs harvested, and 3,476 (71.2\%) patients were staged as ypN0. In the ypN0 group, 38.0\% $(1,322 / 3,476)$ of patients had $<12$ TLNs harvested, and in the ypN+ group, $28.2 \%(396 / 1,405)$ of patients had $<12$ TLNs harvested. CSS was significantly different between patients with $<10$ NLNs and patients with $\geq 10$ NLNs $(\mathrm{P}<0.001)$. Additionally, CSS was not significantly different between patients with $<12$ TLNs harvested and patients and those with $\geq 12$ TLNs harvested (85.6\% vs. 86.4\%, P>0.05).

The ypN stage, LNR and LODDS classification were significantly associated with CSS between subgroups in the total SEER cohort and in patients with $\geq 12$ TLNs harvested $(\mathrm{P}<0.01$ across all groups; Figure $4 A, B, C, D, E, F)$. Similar to the results from our independent cohort, no significant difference was observed between the ypN1 and ypN2 subgroups or between the LNR1 and LNR2 subgroups when the number of TLNs harvested was $<12$ (Figure 4G,H). The LODDS was also significantly associated 
Table 3 Prognostic value of the pathological factors among the $<12$ and $\geq 12$ TLN subgroups

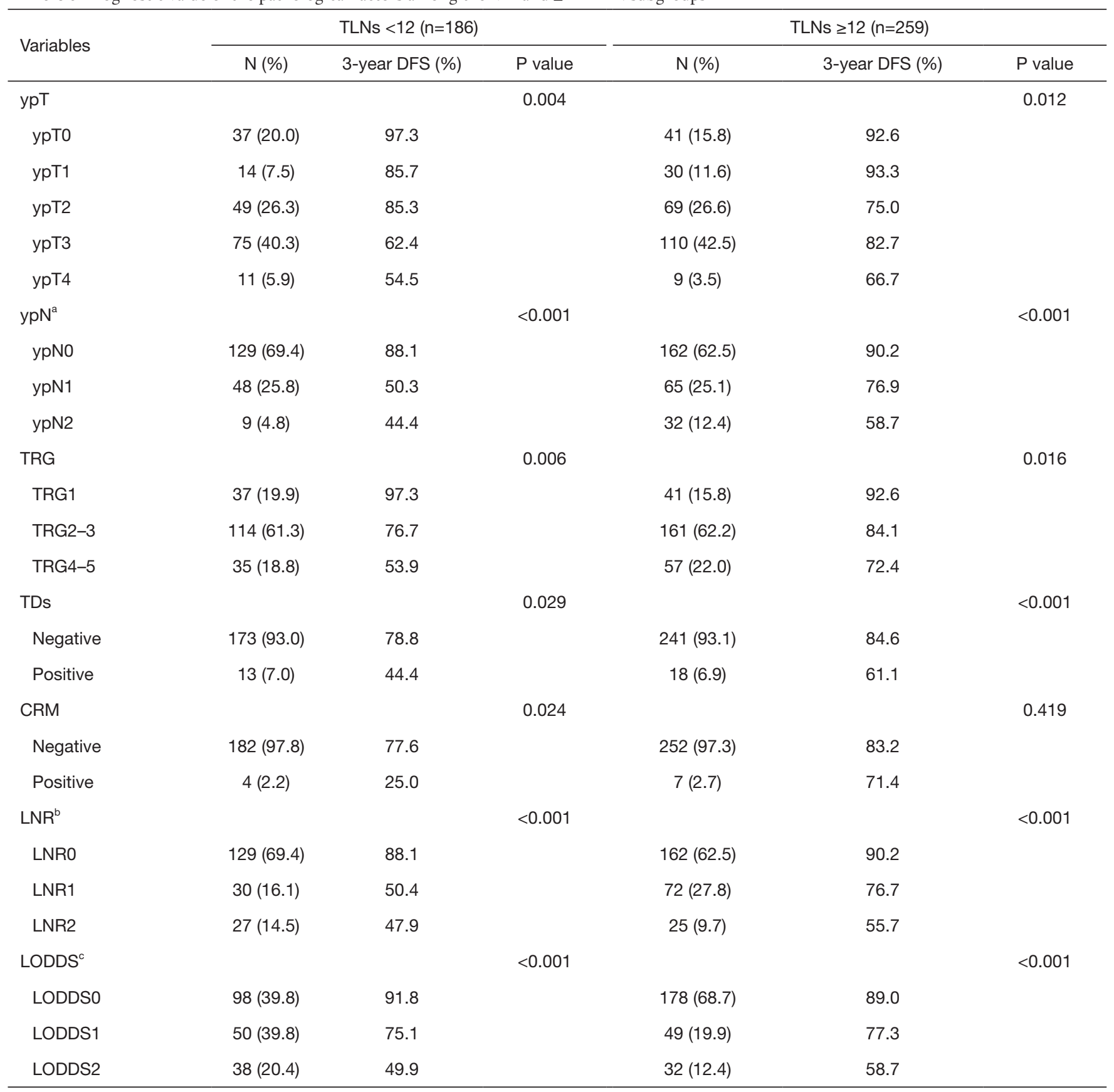

a, $\mathrm{P}<0.05$ across all groups for $y p N$ in TLN $\geq 12$ group; ${ }^{b}, \mathrm{P}<0.05$ across all groups for LNR in TLN $\geq 12$ group; ${ }^{\mathrm{c}}, \mathrm{P}<0.05$ across all groups for LODDS in TLN $\geq 12$ group. TLNs, total number of retrieved lymph nodes; DFS, disease-free survival; TRG, tumor regression grading; TDs, tumor deposits; CRM, circumferential resection margin; LNR, lymph node ratio; LODDS, logarithmic odds of positive lymph nodes.

with CSS when the number of TLNs harvested was $<12$ $(\mathrm{P}<0.01$ across all groups; Figure $4 I)$. We also divided the ypN0 and ypN+ stage patients into different prognostic subgroups according to the LODDS classification system
$(\mathrm{P}<0.05$ across all groups; Figure $5 A, B)$.

The TimeROC analysis revealed a higher AUC for the LODDS classification than for the current ypN staging system or the LNR classification in all patients and in 
A

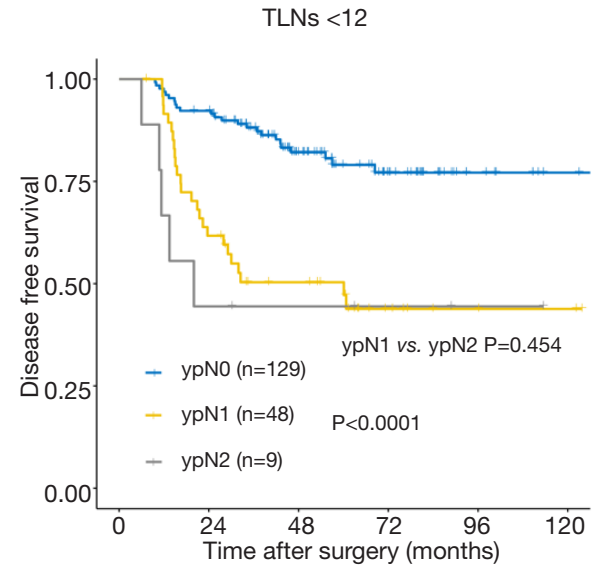

C

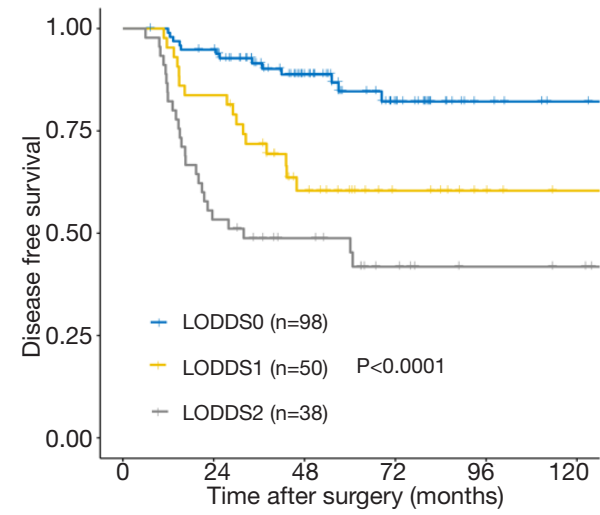

B $\quad$ TLNs $<12$

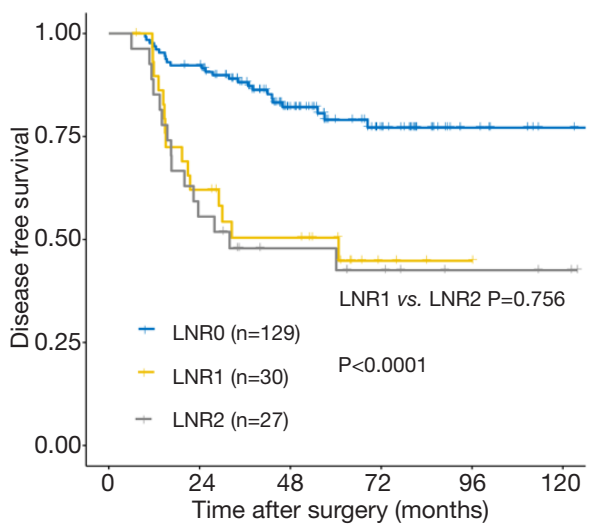

TLNs $<12$

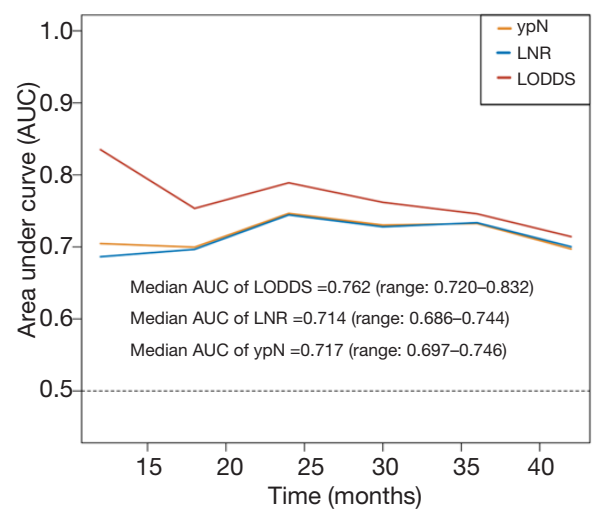

Figure 2 Kaplan-Meier analysis of DFS according to the ypN stage, LNR, and LODDS classification in patients with $<12$ TLNs harvested $(\mathrm{A}, \mathrm{B}, \mathrm{C})$ and TimeROC analysis of the ypN, LNR, and LODDS classification in patients with $<12$ TLNs harvested (D). DFS, disease-free survival; LNR, lymph node ratio; LODDS, logarithmic odds of positive lymph nodes; TLN, total lymph node; TimeROC, time-dependent area under the receiver operating characteristic curve.

the $<12$ TLNs harvested, ypN0, or ypN+ subgroups (Figure 6A,B,C,D). Moreover, LODDS was found to be an independent prognostic factor for $\mathrm{CSS}(\mathrm{P}<0.001, \mathrm{HR}=2.61$, 95\% CI: 2.29-2.98).

\section{Discussion}

As shown in the present study, the ypN stage failed to stratify the patients according to prognosis, partially due to the reduced number of TLNs harvested. Furthermore, the LODDS was a better prognostic factor for DFS than either the current AJCC ypN staging system or the LNR-based approach in patients with LARC after NCRT, particularly those with TLNs $<12$ or with no lymph node metastasis.

Current guidelines recommend a minimum of 12 lymph nodes be harvested from patients with CRC to avoid staging migration (19). However, many studies have revealed that NCRT decreases the number of lymph nodes harvested and then exerts a downstaging effect $(17,20)$. According to one meta-analysis, NCRT leads to a mean reduction in the number of TLNs and PLNs harvested (3.9 and 0.7, respectively) compared to patients without NCRT (4). As shown in the present study, $41.8 \%$ of patients $(n=186)$ had $<12$ TLNs harvested in our cohort, and $35.2 \%$ patients $(\mathrm{n}=1,718)$ had $<12$ TLNs harvested in the SEER validation cohort. In other studies, approximately $30-50 \%$ of the patients had fewer than 12 lymph nodes harvested $(3,6)$. Patients with rectal cancer who are treated with NCRT may have a better prognosis if more than 12 lymph nodes are harvested $(6,21)$. However, one meta-analysis failed to 
A
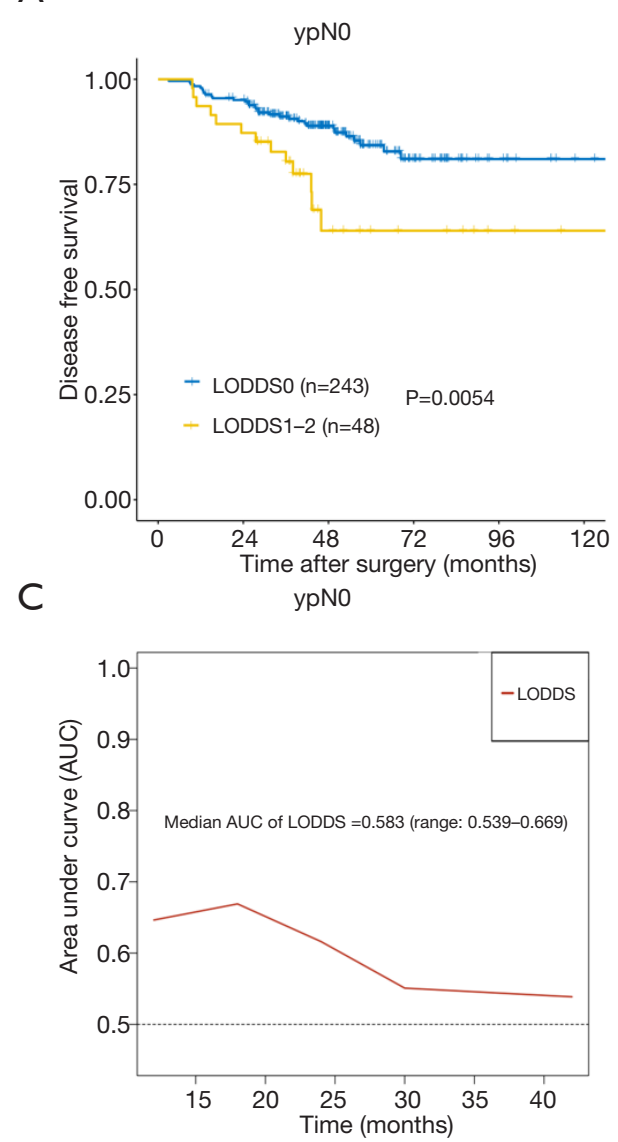

B
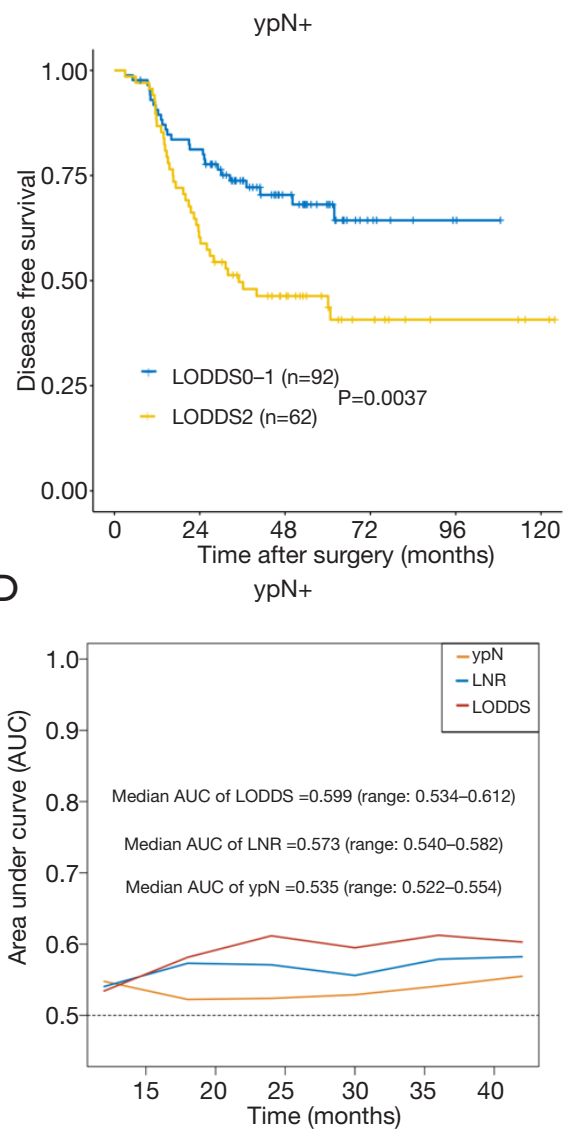

Figure 3 Kaplan-Meier analysis of DFS in the ypN0 and ypN+ subgroups according to the LODDS classification (A,B) and TimeROC analysis of the ypN0 and ypN+ subgroups according to the LODDS classification (C,D). DFS, disease-free survival; LODDS, logarithmic odds of positive lymph nodes; TimeROC, time-dependent area under the receiver operating characteristic curve.

Table 4 Three-year DFS of each ypN stage according to the LODDS classification

\begin{tabular}{|c|c|c|c|c|c|c|c|}
\hline ypN & LODDS0, n (\%) & 3-year DFS (\%) & LODDS1, n (\%) & 3-year DFS (\%) & LODDS2, n (\%) & 3-year DFS (\%) & P value* \\
\hline ypN+ & 33 (21.4) & 71.2 & 59 (38.3) & 62.0 & $62(40.3)$ & 47.5 & 0.032 \\
\hline$P$ value ${ }^{\&}$ & & 0.081 & & 0.570 & & 0.525 & \\
\hline
\end{tabular}

*, comparison of the 3-year DFS between different LODDS groups; ${ }^{\text {}}$, comparison of the 3-year DFS between different ypN groups. DFS, disease-free survival; LODDS, logarithmic odds of positive lymph nodes.

identify a significant association between a cutoff level of 12 lymph nodes harvested and overall survival and/or DFS (4). Han et al. recently reported that health care providers should aim to retrieve 8 or more lymph nodes to enable accurate staging for rectal cancer in patients who received NCRT (22). Therefore, the recommendations to acquire at least 12 lymph nodes from NCRT-treated patients have been disputed. In our study, when the TLN cutoff value was 12, DFS was not significantly different. However, DFS differed according to TLNs when the cutoff value was set to 7. Therefore, the rule of acquiring at least 12 lymph nodes is not an acknowledged standard in NCRT-treated patients, 
A

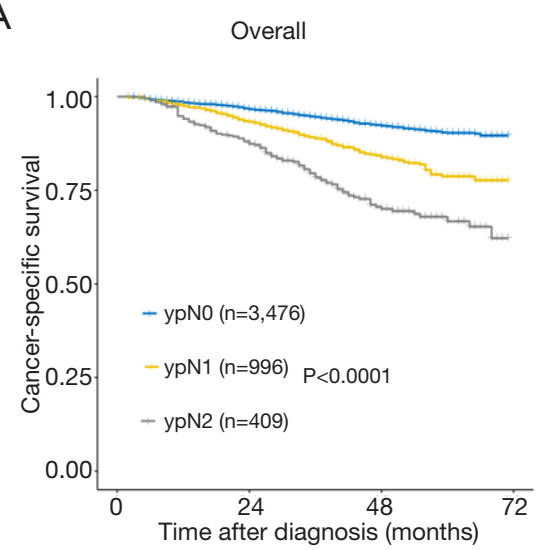

D

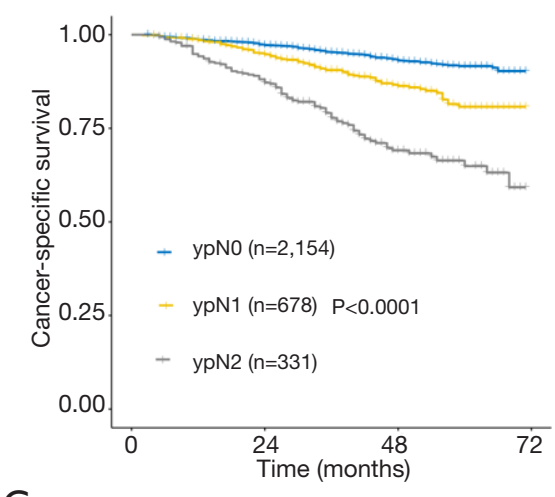

G

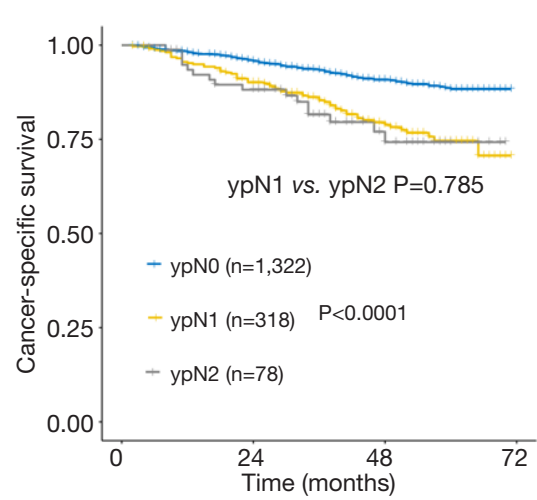

B

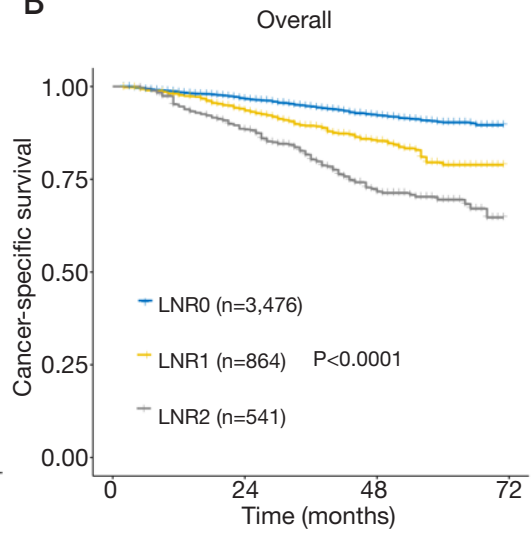

$\mathrm{E}$

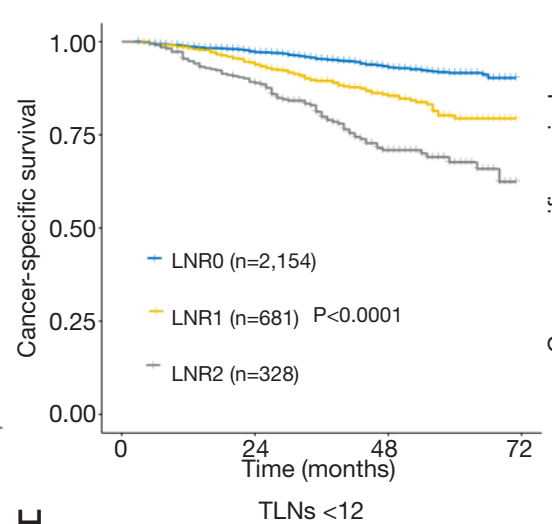

$\mathrm{H}$

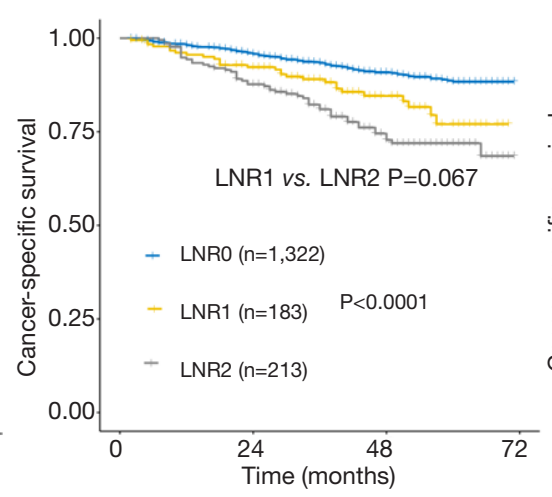

C

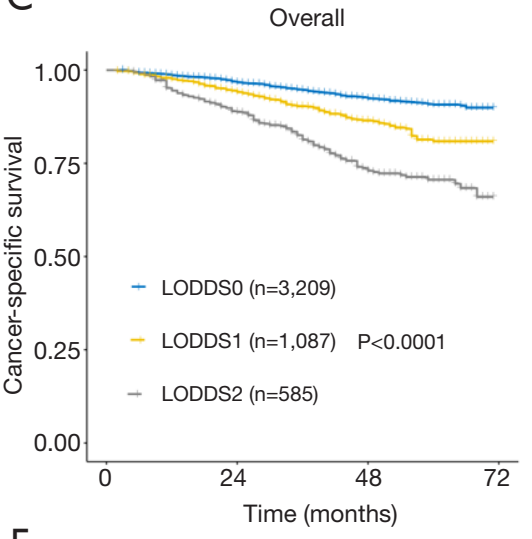

$\mathrm{F}$

TLNs $\geq 12$
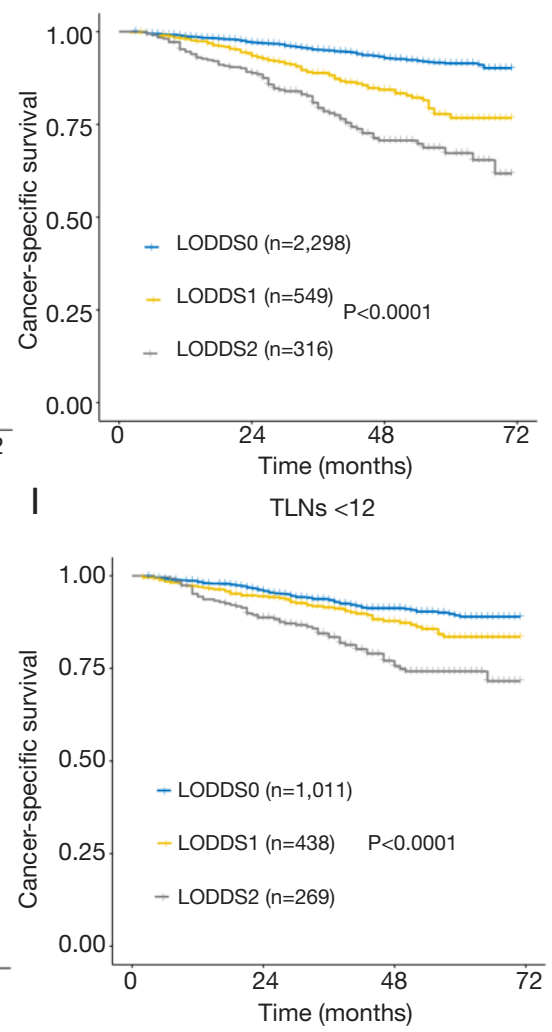

Figure 4 Kaplan-Meier analysis of CSS according to the ypN, LNR and LODDS classification for all patients (A,B,C), those in the $\geq 12$ TLNs (D,E,F) subgroup, and those in the $<12$ TLNs (G,H,I) subgroup in the SEER validation cohort. CSS, cancer-specific survival; LNR, lymph node ratio; LODDS, logarithmic odds of positive lymph nodes; TLN, total lymph node; SEER, Surveillance, Epidemiology and End Results.

and the most suitable number of lymph nodes requires further research.

NCRT often not only leads to fewer TLNs and PLNs harvested but also results in ypN stage migration (4). Thus, the prognostic value of ypN staging may be diminished.
According to Park et al. and Wang et al., the ypN category cannot be used to divide patients into prognostic groups $(6,23)$. Our study did not reveal a significant difference in DFS between the ypN1 group and the ypN2 group, suggesting that $\mathrm{ypN}$ staging failed to satisfactorily stratify 
A

ypNO

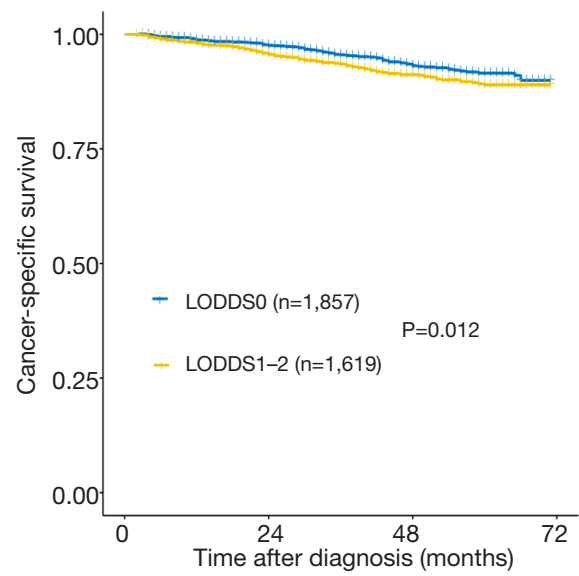

B

$\mathrm{ypN}+$

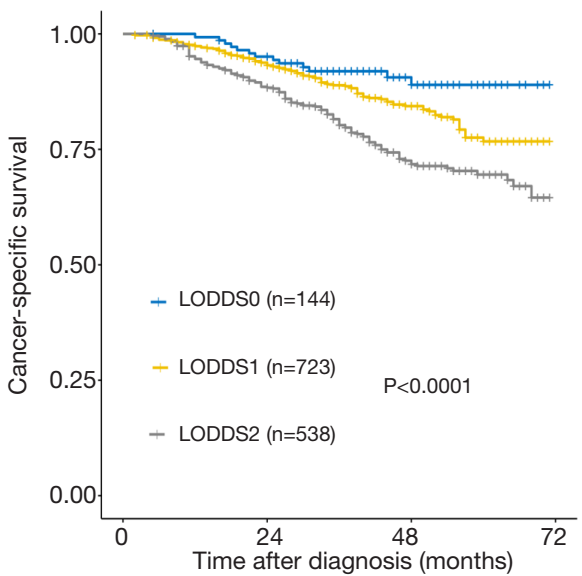

Figure 5 Kaplan-Meier analysis of CSS in the in the ypN0 subgroup (A) and ypN+ subgroup (B) according to the LODDS classification in the SEER validation cohort. CSS, cancer-specific survival; LODDS, logarithmic odds of positive lymph nodes; SEER, Surveillance, Epidemiology and End Results.

the patients. Interestingly, the differences in prognosis were statistically significant in patients stratified according to the ypN staging system when $\geq 12$ TLNs were harvested. However, ypN staging failed to satisfactorily stratify the patients into ypN1 and ypN2 subgroups when $<12$ TLNs were harvested. Similar to the results from our independent cohort, the ypN staging system was not significantly associated with CSS in the ypN1 and ypN2 groups from the SEER cohort when TLNs $<12$. The prognosis determined using ypN staging was significantly different from that using the total cohort of SEER patients, which may be due to the smaller percentage of patients in the SEER cohort with $<12$ TLNs harvested than in our cohort (35.2\% vs. 41.8\%). Based on these results, ypN staging failed to satisfactorily stratify the patients, partially due to the reduced number of TLNs harvested.

Some authors have introduced the LNR, which combines TLNs with PLNs, to overcome the limitation posed by harvesting fewer TLNs and to improve the prognostic value of ypN staging. The LNR has served as a novel prognostic factor in various cancer types $(20,23)$. As shown in a recent study by Klos et al., the LNR has a stronger prognostic value than the absolute number of PLNs (20). In our study, the LNR classification system was significantly associated with DFS for the total patient cohort. However, the LNR was not associated with DFS in the comparison of LNR1 and LNR2 subgroups when $<12$ TLNs were harvested. Moreover, similar to our cohort, the LNR classification system was not significantly associated with CSS in the LNR1 and LNR2 groups of the SEER cohort when $<12$ TLNs were harvested. In addition, the prognostic value of the LNR is limited; first, it is unable to predict the prognosis of ypN0 stage patients, and second, the LNR is not useful when the number of PLNs is equal to the number of TLNs retrieved. Therefore, a better lymph node staging system is needed to overcome these disadvantages.

The LODDS classification has been recently described as a predictive factor for lymph node staging, which may improve the prognostic accuracy of $\mathrm{pN}$ classification and serve as a better prognostic factor than LNR and $\mathrm{pN}(9,10,13,14)$. Huang et al. revealed the superior discriminatory power of LODDS to LNR in terms of determining the CSS of the ypN+ group (13). However, this study was only based on data from the SEER public database and was not validated in another cohort. Moreover, the study only studied the prognostic role of the LODDS in patients with stage III tumors. After neoadjuvant therapy, more than $60 \%$ of the patients had no lymph node metastasis, and the proportion of patients with $<12$ TLNs harvested was higher in the ypN0 group than in the ypN+ group. Neither the LNR nor ypN staging system provided additional prognostic information for patients with ypN0 stage and $<12$ TLNs were harvested, while the LODDS considers the effects of both the numbers of PLNs and NLNs on prognosis; thus, it may provide more accurate prognostic information for patients, particularly those with 
A

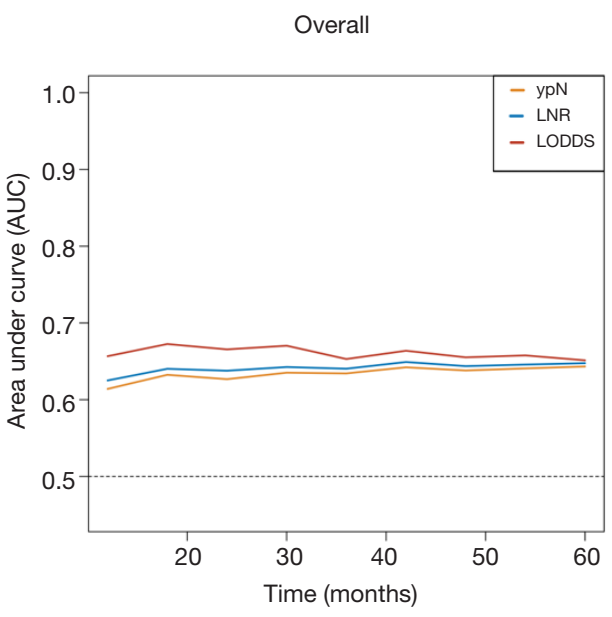

C

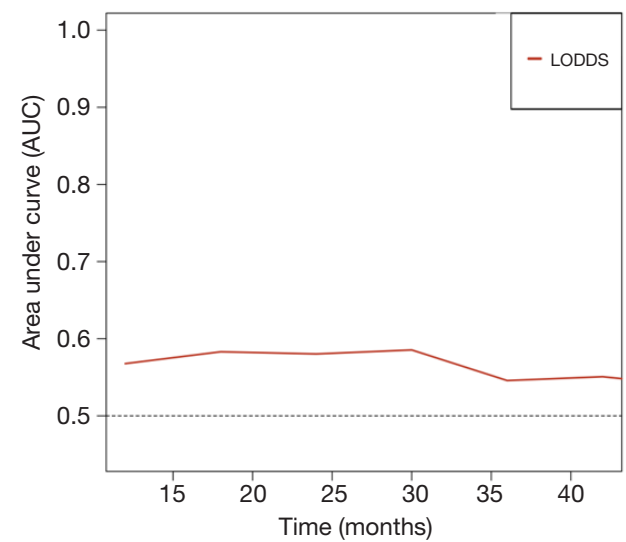

B

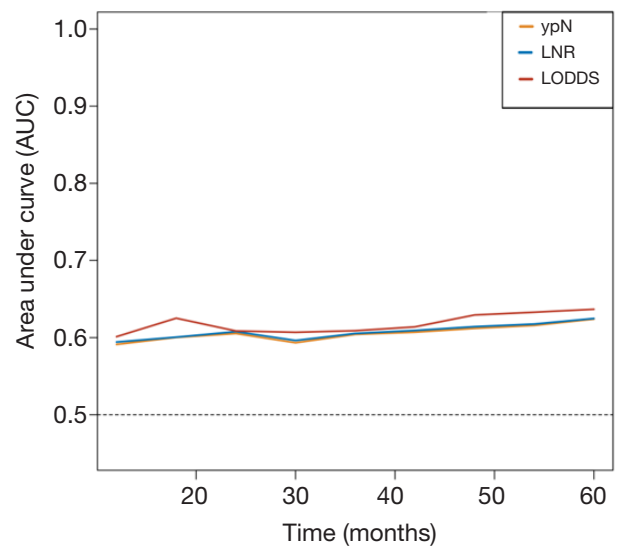

D $\quad$ ypN+

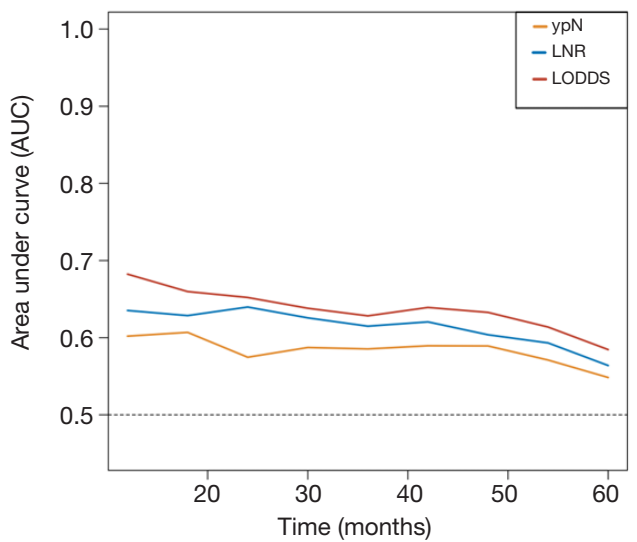

Figure 6 TimeROC analysis of the ypN, LNR and LODDS classification for all patients (A), the $<12$ TLNs subgroup (B), the ypN0 subgroup (C), and the ypN+ subgroup (D), respectively, in the SEER validation cohort. TimeROC, time-dependent area under the receiver operating characteristic curve; LNR, lymph node ratio; LODDS, logarithmic odds of positive lymph nodes; TLN, total lymph node; SEER, Surveillance, Epidemiology and End Results.

stage ypN0 disease or $<12$ TLNs harvested.

In our study, the LODDS classification system was significantly associated with DFS, and the LODDS could subclassify ypN0 stage patients into a high-risk group and a low-risk group. Furthermore, the LODDS classification system was significantly associated with DFS, regardless of whether $<12$ or $\geq 12$ TLNs were harvested. Moreover, the TimeROC analysis revealed that the LODDS classification had a higher AUC for DFS than the current ypN staging system or the LNR classification for the overall cohort, those with $<12$ TLNs harvested, or ypN0 or ypN+ subgroups, respectively. The results of the multivariate analysis showed that the LODDS classification was an independent prognostic factor. The data from the SEER database confirmed these results. Thus, the LODDS classification is a better prognostic factor and has better accuracy in determining patient prognosis than either ypN staging or the LNR for patients with rectal cancer treated with NCRT, particularly those with TLNs $<12$ and those with no lymph node metastasis.

To the best of our knowledge, this study is the first to show that ypN staging failed to satisfactorily stratify patients treated with NCRT, partially when few TLNs are harvested. The LODDS classification considers the effects of both PLN and NLN numbers on patient prognosis and minimizes the bias associated with the number of TLNs 
harvested. Therefore, the LODDS classification has a natural advantage in evaluating patients without lymph node metastases. As shown in the present study, the LODDS is a better independent prognostic factor than ypN and LNR for patients with LARC who underwent NCRT, particularly for patients with $<12$ TLNs harvested or those with no lymph node metastasis, and it is expected to have general value for patients with other types of cancers.

Our study has several limitations that should be considered when interpreting the results. First, this study was retrospective in nature and included a relatively limited number of patients from only one hospital, although we did confirm our results using patients from the SEER database. Our findings therefore must still be verified in a multicenter study with larger cohorts. Second, the chemotherapeutic regimens administered to the patients were heterogeneous, as only half of the patients received postoperative adjuvant chemotherapy.

In conclusion, compared with the current AJCC ypN staging system and the LNR-based approach, the LODDS classification was a better independent prognostic factor for patients with LARC who underwent NCRT, particularly those who had $<12$ harvested or no lymph node metastasis.

\section{Acknowledgments}

Funding: This study was partially supported by grants from the CAMS Innovation Fund for Medical Sciences (CIFMS; 2016-I2M-1-007), CAMS Innovation Fund for Medical Sciences (CIFMS; 2019-I2M-1-003) and National Natural Science Foundation of China (81972317).

\section{Footnote}

Reporting Checklist: The authors have completed the REMARK reporting checklist. Available at http://dx.doi. org/10.21037/atm-20-7590

Data Sharing Statement: Available at http://dx.doi. org/10.21037/atm-20-7590

Peer Review File: Available at http://dx.doi.org/10.21037/ atm-20-7590

Conflicts of Interest: All authors have completed the ICMJE uniform disclosure form (available at http://dx.doi. org/10.21037/atm-20-7590). The authors have no conflicts of interest to declare.
Ethical Statement: The authors are accountable for all aspects of the work in ensuring that questions related to the accuracy or integrity of any part of the work are appropriately investigated and resolved. All procedures performed in this study involving human participants were in accordance with the Declaration of Helsinki (as revised in 2013). This study was reviewed and approved by the Ethics Committee of Cancer Hospital, Chinese Academy of Medical Sciences (No. NCC2019C-096). Because of the retrospective nature of the study, the requirement for informed consent was waived.

Open Access Statement: This is an Open Access article distributed in accordance with the Creative Commons Attribution-NonCommercial-NoDerivs 4.0 International License (CC BY-NC-ND 4.0), which permits the noncommercial replication and distribution of the article with the strict proviso that no changes or edits are made and the original work is properly cited (including links to both the formal publication through the relevant DOI and the license). See: https://creativecommons.org/licenses/by-nc-nd/4.0/.

\section{References}

1. Gérard JP, Azria D, Gourgou-Bourgade S, et al. Clinical outcome of the ACCORD 12/0405 PRODIGE 2 randomized trial in rectal cancer. J Clin Oncol 2012;30:4558-65.

2. Doescher J, Veit JA, Hoffmann TK. The 8th edition of the AJCC Cancer Staging Manual: updates in otorhinolaryngology, head and neck surgery. $\mathrm{HNO}$ 2017;65:956-61.

3. Kim J, Huynh R, Abraham I, et al. Number of lymph nodes examined and its impact on colorectal cancer staging. Am Surg 2006;72:902-5.

4. Mechera R, Schuster T, Rosenberg R, et al. Lymph node yield after rectal resection in patients treated with neoadjuvant radiation for rectal cancer: a systematic review and meta-analysis. Eur J Cancer 2017;72:84-94.

5. Kim HJ, Jo JS, Lee SY, et al. Low lymph node retrieval after preoperative chemoradiation for rectal cancer is associated with improved prognosis in patients with a good tumor response. Ann Surg Oncol 2015;22:2075-81.

6. Wang Y, Zhou M, Yang J, et al. Increased lymph node yield indicates improved survival in locally advanced rectal cancer treated with neoadjuvant chemoradiotherapy. Cancer Med 2019;8:4615-25.

7. Amajoyi R, Lee Y, Recio PJ, et al. Neoadjuvant therapy 
for rectal cancer decreases the number of lymph nodes harvested in operative specimens. Am J Surg 2013;205:28992; discussion 292.

8. Govindarajan A, Gonen M, Weiser MR, et al. Challenging the feasibility and clinical significance of current guidelines on lymph node examination in rectal cancer in the era of neoadjuvant therapy. J Clin Oncol 2011;29:4568-73.

9. Wang J, Hassett JM, Dayton MT, et al. The prognostic superiority of log odds of positive lymph nodes in stage III colon cancer. J Gastrointest Surg 2008;12:1790-6.

10. Ramacciato G, Nigri G, Petrucciani N, et al. Prognostic role of nodal ratio, LODDS, $\mathrm{pN}$ in patients with pancreatic cancer with venous involvement. BMC Surg 2017;17:109.

11. Dinaux AM, Leijssen L, Bordeianou LG, et al. Outcomes of persistent lymph node involvement after neoadjuvant therapy for stage III rectal cancer. Surgery 2018;163:784-8.

12. Sun $Y$, Zhang $Y$, Huang $Z$, et al. Prognostic implication of negative lymph node count in ypN+ rectal cancer after neoadjuvant chemoradiotherapy and construction of a prediction nomogram. J Gastrointest Surg 2019;23:1006-14.

13. Huang B, Ni M, Chen C, et al. LODDS is superior to lymph node ratio for the prognosis of node-positive rectal cancer patients treated with preoperative radiotherapy. Tumori 2017;103:87-92.

14. Scarinci A, Di Cesare T, Cavaniglia D, et al. The impact of $\log$ odds of positive lymph nodes (LODDS) in colon and rectal cancer patient stratification: a single-center analysis of 323 patients. Updates Surg 2018;70:23-31.

15. Sauer R, Becker H, Hohenberger W, et al. Preoperative versus postoperative chemoradiotherapy for rectal cancer. N Engl J Med 2004;351:1731-40.

16. Sauer R, Liersch T, Merkel S, et al. Preoperative versus postoperative chemoradiotherapy for locally advanced

Cite this article as: $\mathrm{Xu}$ T, Zhang L, Yu L, Zhu Y, Fang H, Chen B, Zhang H. Log odds of positive lymph nodes is an excellent prognostic factor for patients with rectal cancer after neoadjuvant chemoradiotherapy. Ann Transl Med 2021;9(8):637. doi: $10.21037 / \mathrm{atm}-20-7590$ rectal cancer: results of the German CAO/ARO/AIO-94 randomized phase III trial after a median follow-up of 11 years. J Clin Oncol 2012;30:1926-33.

17. Gurawalia J, Dev K, Nayak SP, et al. Less than 12 lymph nodes in the surgical specimen after neoadjuvant chemoradiotherapy: an indicator of tumor regression in locally advanced rectal cancer? J Gastrointest Oncol 2016;7:946-57.

18. Mandard AM, Dalibard F, Mandard JC, et al. Pathologic assessment of tumor regression after preoperative chemoradiotherapy of esophageal carcinoma. Clinicopathologic correlations. Cancer 1994;73:2680-6.

19. Benson AB 3rd, Venook AP, Al-Hawary MM, et al. Rectal cancer, version 2.2018, NCCN Clinical Practice Guidelines in Oncology. J Natl Compr Canc Netw 2018;16:874-901.

20. Klos CL, Bordeianou LG, Sylla P, et al. The prognostic value of lymph node ratio after neoadjuvant chemoradiation and rectal cancer surgery. Dis Colon Rectum 2011;54:171-5.

21. Abdel-Misih SR, Wei L, Benson AB 3rd, et al. Neoadjuvant therapy for rectal cancer affects lymph node yield and status without clear implications on outcome: the case for eliminating a metric and using preoperative staging to guide therapy. J Natl Compr Canc Netw 2016;14:1528-34.

22. Han J, Noh GT, Yeo SA, et al. The number of retrieved lymph nodes needed for accurate staging differs based on the presence of preoperative chemoradiation for rectal cancer. Medicine (Baltimore) 2016;95:e4891.

23. Park IJ, Yu CS, Lim SB, et al. Ratio of metastatic lymph nodes is more important for rectal cancer patients treated with preoperative chemoradiotherapy. World J Gastroenterol 2015;21:3274-81. 